The Social Sciences 14 (11): 370-374, 2019

ISSN: $1818-5800$

(C) Medwell Journals, 2019

\title{
Brain Wave and Consumer's Choice in Response to Advertisement
}

\author{
Norsiah Fauzan and Sandhesa Rajendran \\ Faculty of Cognitive Science and Human Development, University Malaysia Sarawak, \\ 94300 Kota Samarahan, Malaysia
}

\begin{abstract}
An assessment of brain wave on mind behavior of consumers from six participants were conducted while watching advertisement stimuli with two brands of milk powder (Enfagrow and Dugrow). Six participants volunteered for the study and their brain waves were recorded to measure their frequencies during their engagement and decision making. The analysis showed higher activity of beta and alpha were stimulated while watching Enfagrow, Dugro advertisement stimulated higher theta in the frontal area and temporal area, especially, at T6-Av with $\left(19.83 \mathrm{uV}^{2}\right)$ amplitude compared to Enfagrow advertisement with $\left(2.99 \mathrm{uV}^{2}\right)$ amplitude.
\end{abstract}

Key words: Brain wave, consumer's choice, QEEG, milk powder, temporal area, advertisement

\section{INTRODUCTION}

Research in consumer's decision making has benefitted from the revealed preferences perspective that follows the tradition of focusing on observation on what people actually choose or using questionnaire and interview to get their reasoning for their choice (Fauzan, 2015). Most marketers are using verbal measures on pre-test TV advertisements due to the limitation to provide effective measures on internal reaction towards the stimuli ignoring mechanisms inside "the black box" in which the decisions were made in relation to other additional variables such as stress, intentions, attitudes and memory. These biological variables in neuroscience and its interactions with sociological variables adds value to decision making and consumer choice research. Since, 2012, the neuromarketing companies are increasing from 13-60 companies (Plassmann et al., 2008).

Neuroscience based research using neuroimaging tools such as fMRI or Quantitative Electroencephalograph (QEEG) is an added advantage to show what's going on inside the brain and not making baseless advertising claims in marketing.

In previous research, the commonly used device to look at the neuromechanism such as emotions and memory in response to advertisement is fMRI conducted in a medical environment where it requires them to lie inside a machine that can be intimidating. In this research, QEEG tool were used to give description of neural mechanism that associated with advertisement effects or stimuli. EEG is portable and can easily record the brain activity with the cap of electrodes placed on the surface of your head, however, the recording did not get to the deep areas of the brain.
Objective: The objective of this study is to describe neural mechanism of consumer behaviour in response to advertisement to identify the dominant waves at specific regions of the brain when consumer is engaged watching advertisement stimuli and to determine the aspects that influence consumer's choice.

\section{MATERIALS AND METHODS}

Research design and procedure: This is an observation study using Quantitative Electroencephalogram (QEEG) to record the brainwaves of six volunteered subjects consisting of mothers within the range of 23-45 years old. The subjects were observed during the eyes opened, eyes closed task and decision making process. During the decision making process the subjects were watching two commercial advertisements on milk powder while their brainwaves were recorded. The participants were observed and interviewed to obtain their correlated behaviour towards advertising stimuli.

Instruments: The instrument used in this research was QEEG tool, two commercial advertisement, observation and interviews. Before this study was conducted, the participants were explained on how the cognitive task will be done and the data obtained were to be kept as confidential. Two commercial advertisements were chosen as video stimulus while the brain wave pattern of the participants were recorded. Both ads are regarding children's milk powder but with different brands and pricing. The brands are Dumex Dugro (emotional inclined) and Enfagrow (brain science knowledge) where the duration of the former adverstisement was $1 \mathrm{~min}$ and 
$3 \mathrm{sec}$ whereas the later were $30 \mathrm{sec}$. The report were graphically represented in colour brain maps, EEG power spectra graph and EEG power spectra table with different sub-bands.

Interview and observation: Observation were done during the QEEG brain mapping process whereas the interview was conducted after the six participants went through the QEEG brain mapping process. Questions asked includes product preferences and why they have chosen the product.

\section{RESULTS AND DISCUSSION}

The raw data were analyzed thoroughly and the result were keyed in excel master sheet for further analysis on the brainwaves from different bands and sub bands.

Eyes opened task: In the eyes opened task, the beta wave has the average value of $22.21 \mathrm{uV}^{2}$ amplitude which shows that the respondents are in the state of focused and has high attention in the required task (Fig. 1-3).
The differences of frequency of brainwaves pattern using QEEG single electrode beta waves: In the first analysis, it was observed that Enfagrow advertisement has bigger beta activity on the FP1 area with the average value of $4.64 \mathrm{uV}^{2}$ amplitude as compared to the Dugro advertisement which has the average value of $3.72 \mathrm{uV}^{2}$.

The differences of frequency of brainwaves pattern using QEEG single electrode beta waves: In the first analysis, it was observed that Enfagrow advertisement has bigger beta activity on the FP1 area with the average

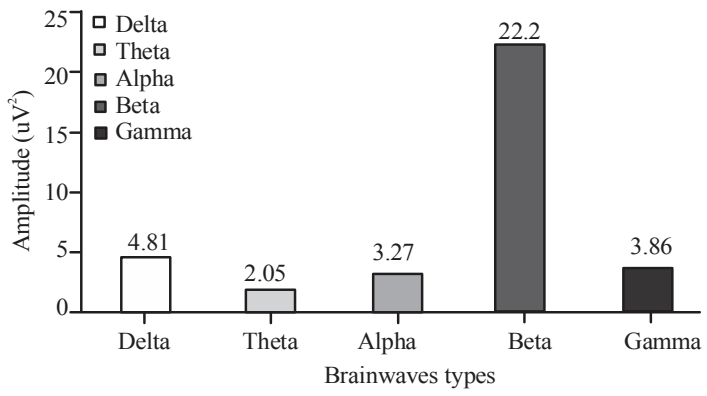

Fig. 1: Average brainwaves recording values of all frequency bands for eyes opened task
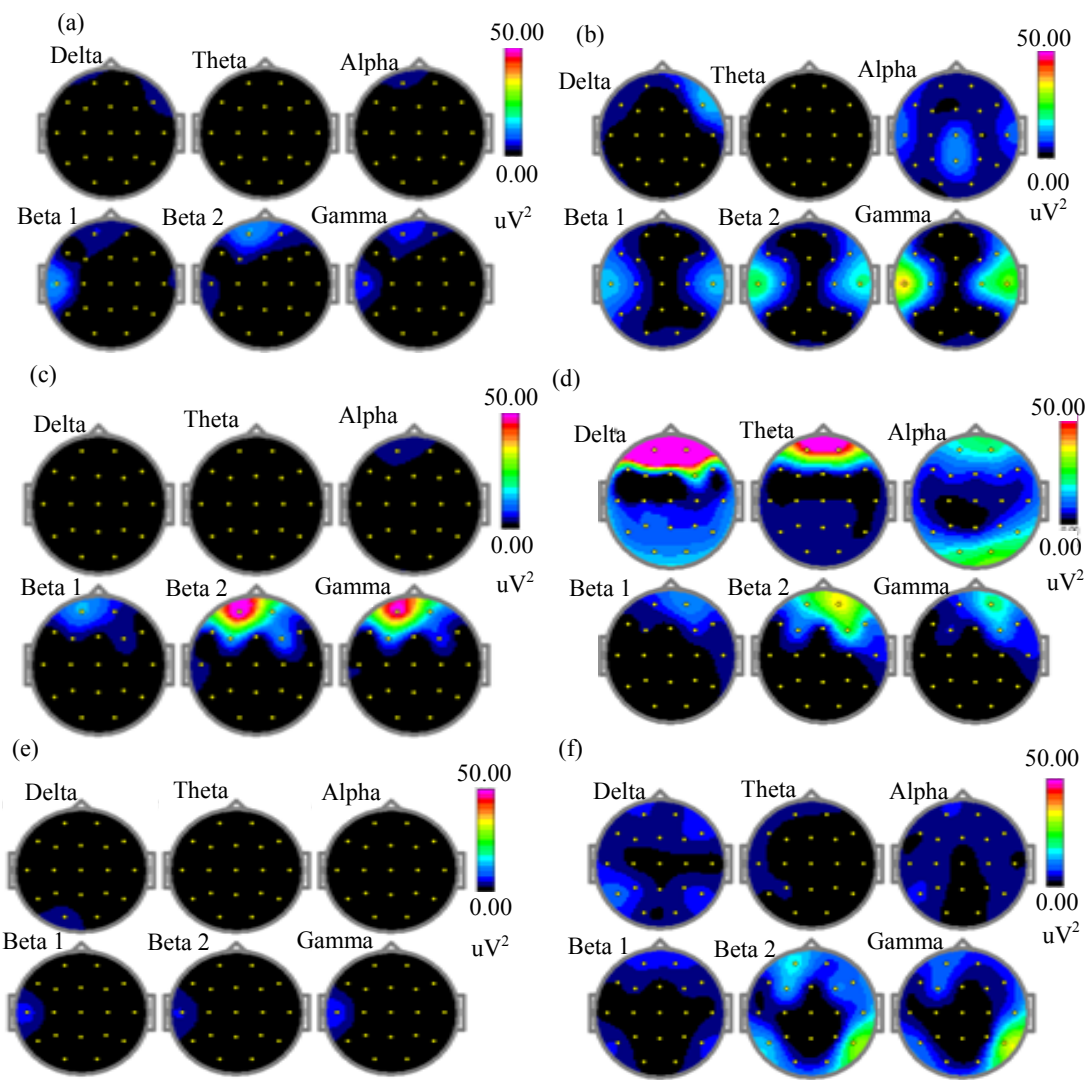

Fig. 2(a-f): Brain topography of all subjects in the eyes opened task 


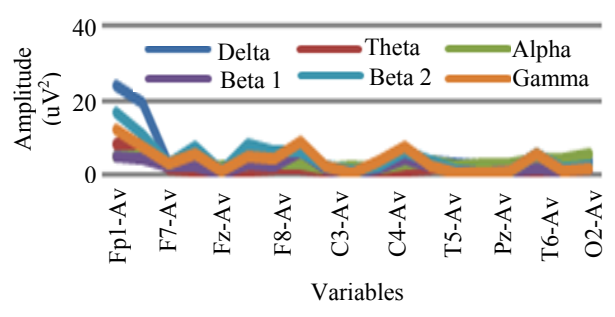

Fig. 3: Average brainwave activities of all respondents across all channels electrode

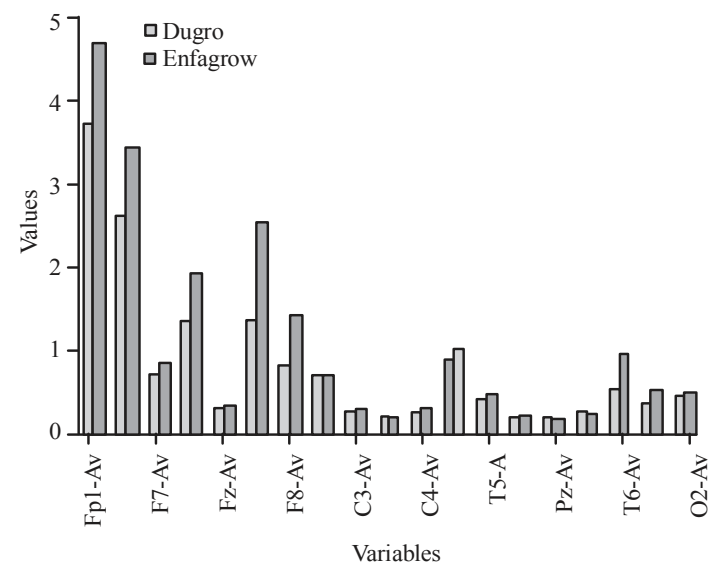

Fig. 4: Beta waves of Dugro and Enfagrow advertisement

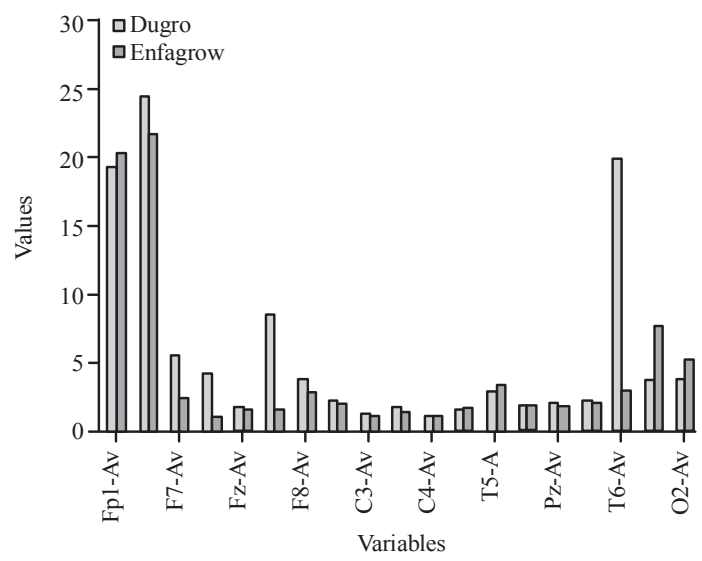

Fig. 5: Theta waves of Dugro and Enfagrow adevertisement

value of $4.64 \mathrm{uV}^{2}$ amplitude as compared to the Dugro advertisement which has the average value of $3.72 \mathrm{uV}^{2}$ amplitude. This shows a high level of focus and attention towards the advertisement. The second highest beta activity was at FP2 area related to judgment. The response to enfagrow advertisement is higher versus Dugro advertisement (Fig. 4).

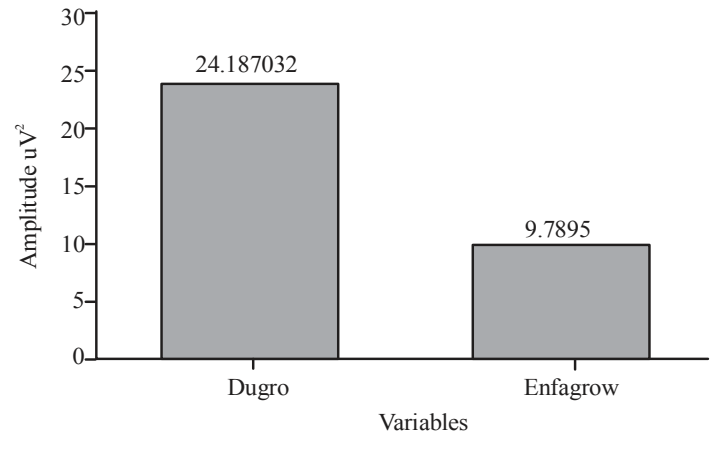

Fig. 6: Theta wave of frontal area for Dugro advertisement

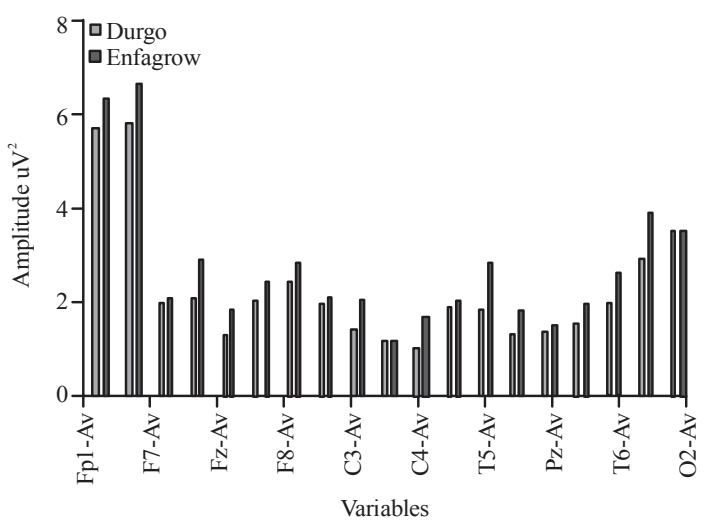

Fig. 7: Alpha waves of Dugro and Enfagrow advertisements

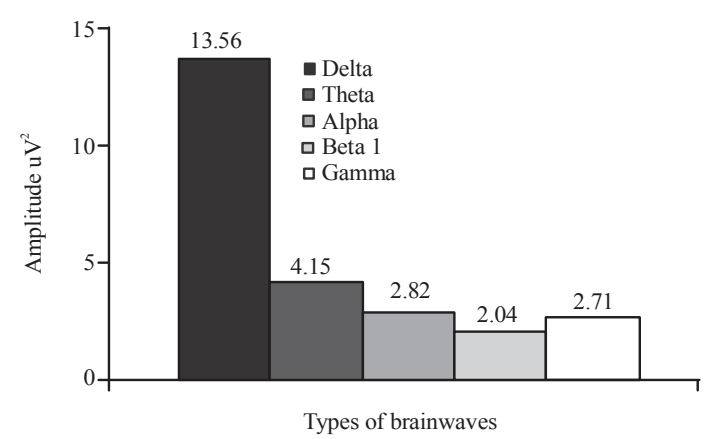

Fig. 8: Average brainwaves recording values of all frequency bands for task 4

Figure 5 revealed a higher theta wave in response to Dugro advertisement in the frontal area and at the temporal area of T6-Av with $\left(19.83 \mathrm{uV}^{2}\right)$ amplitude compared to Enfagrow advertisement with $\left(2.99 \mathrm{uV}^{2}\right)$ amplitude. Nevertheless T6-Av region is associated with emotional understanding. The results implicates a correlation between theta and emotional as suggested by previous researchers (Aftanas and Varlamov, 2005) (Fig. 6-8). 

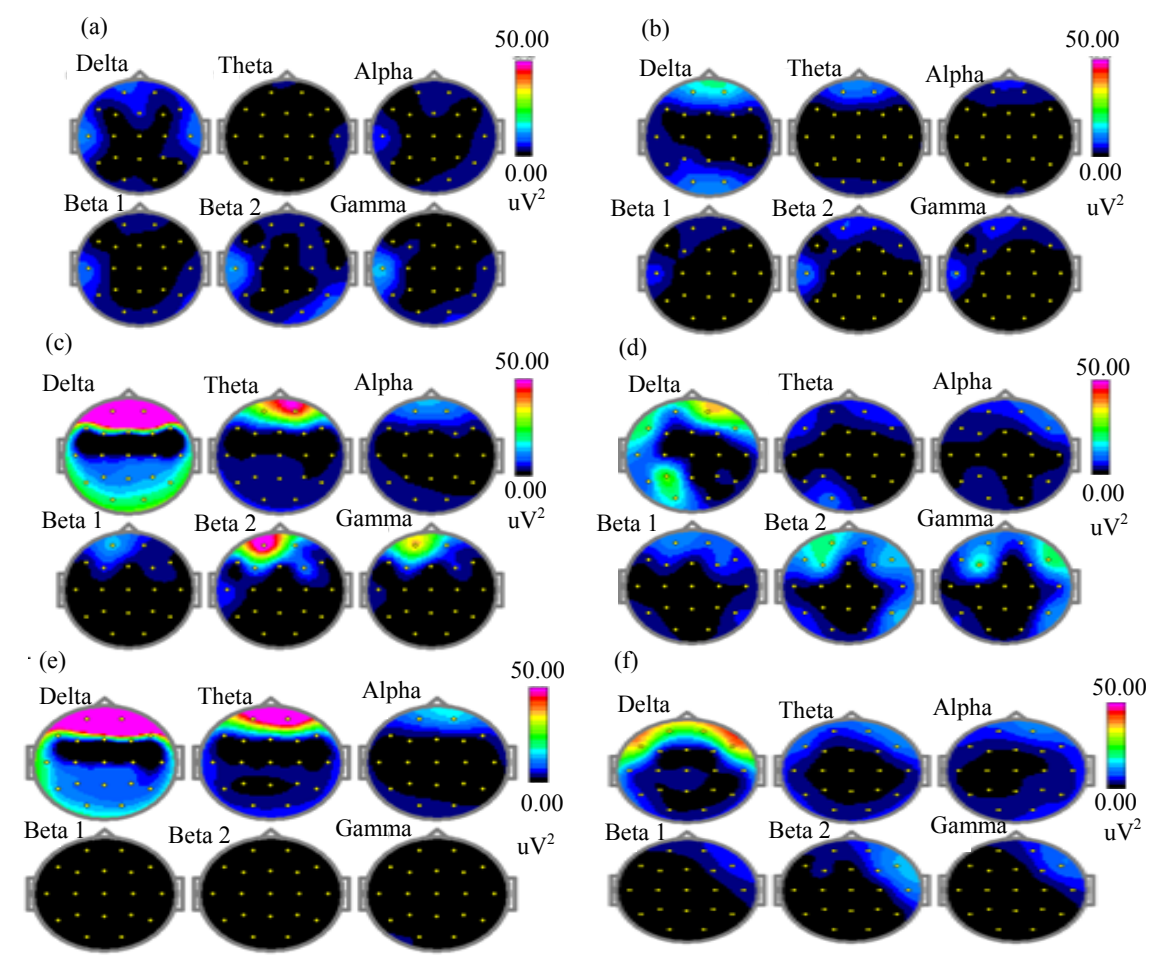

Fig. 9(a-f): Brain topography of all subjects for decision making task

Higher theta activity on the frontal area suggested that Dugro advertisement projected more emotional contents compared to Enfagrow advertisement. Based on Fig. 7 and 8, the dominant brain region for alpha rhythm were on the frontal area and the occipital region. More alpha wave were stimulated at the entire region during Enfagraw advertisement compared to Dugro product. Alpha rhythm is associated with decreasing of information processing. Obviously, the subjects had chosen Dugro based on the emotions (Aftanas and Varlamov, 2005).

Decision making: In decision making task, the dominant brainwaves was delta wave. As shown in Fig. 8, the delta brainwave has the average value of $13.56 \mathrm{uV}^{2}$ amplitude which is the highest brainwave recorded as compared to the other brainwaves obtained from the subjects. The high delta brainwave activity in this task shows that all subjects were in the state of decision making process requiring them to identify products of their choices. In this task much concentration is needed for the subject to think and decide on their choice. Similarly, in Fig. 8 there is high delta wave present at the frontal cortex on most of the subjects.

The primary goal of this study was to describe the neural mechanism of consumer behaviour and the parts of the brain engaging with advertising stimuli related to milk powder. Based on the open eyes task, the results were quite predictable, beta wave (Attention) were dominant, high activation of beta waves indicates that all 6 subjects were in the state of focus throughout this research to ensure the subjects is focused while watching the advertisement. Even though more attention and higher activity of beta and alpha were stimulated while watching Enfagrow, Dugro advertisement stimulated higher theta wave in the frontal area and temporal area, especially, at $\mathrm{T} 6-\mathrm{Av}$ with $\left(19.83 \mathrm{uV}^{2}\right)$ amplitude compared to Enfagrow advertisement with $\left(2.99 \mathrm{uV}^{2}\right)$ amplitude. T6-Av region is associated with emotional understanding that implicates a correlation between theta and emotional processes as suggested by previous researches (Aftanas and Varlamov, 2005). The high beta activity on the left frontal while watching Enfagrow showed a positive response compared to Dugrow that evoked a higher theta wave. This is in line with Davidson's approach-withdrawal theory, the left frontal brain region may be more active toward positive emotional stimuli linked with approach-related behaviors and the right frontal region more active toward negative emotional stimuli linked with withdrawal-related behaviors (Ohme et al., 2010). Thus, the consumers decision and response are indicated by the physiological responses of specific brain waves at different region of the brain such as frontal and temporal region. The results in this research revealed that Enfagrow advertisement implicates on the subjects emotion and 
decision making. Interview from the two participants iterated that their decision making were based on the price of milk powder and the advertisement projecting emotional response. This is in line with the.

\section{CONCLUSION}

The results shows that attention is given more to the second advertisement, there is also activation of alpha rhythm in the occipital cortex as compared to the alpha rhythm for the first advertisement. This could mean that the subjects are not interested when watching the second advertisement (Aftanas and Varlamov, 2005). However, in the interview, two participants iterated that their decision making were based on the price of milk powder and the advertisement projecting emotional response. Beside emotional content, price also plays a vital role in consumer decision making in order to choose the products they favoured (Feige et al., 2005).

\section{ACKNOWLEDGEMENTS}

This research was supported by Faculty of Cognitive Science and Human Development, University Malaysia Sarawak.

\section{REFERENCES}

Aftanas, L.I. and A.A. Varlamov, 2005. Alexithymia impact on EEG activity of anterior and posterior regions of the right hemisphere while experiencing positive and negative emotions. Ross. Fiziol. Zh. Im. I. M. Sechenova, 91: 1121-1131.

Fauzan, N., 2015. Understanding the neuromechanisms of consumer behavior in advertising industry. Eur. J. Econ. Bus. Stud., 1: 149-153.

Feige, B., K. Scheffler, F. Esposito, F. Di Salle, J. Hennig and E. Seifritz, 2005. Cortical and subcortical correlates of electroencephalographic alpha rhythm modulation. J. Neurophysiol., 93: 2864-2872.

Ohme, R., D. Reykowska, D. Wiener and A. Choromanska, 2010. Application of frontal EEG asymmetry to advertising research. J. Econ. Psychol., 31: 785-793.

Plassmann, H., J. O'Doherty, B. Shiv and A. Rangel, 2008. Marketing actions can modulate neural representations of experienced pleasantness. Proc. Natl. Acad. Sci. United States Am., 105: 1050-1054. 